\title{
Commentary \\ Is smaller high enough? Another piece in the puzzle of stress, strain, size, and systems
}

\author{
Michael Quintel and Onnen Moerer
}

Department of Anaesthesia and Intensive Care Medicine, University of Göttingen, Robert Koch Strasse 40, 37075 Göttingen, Germany

Corresponding author: Michael Quintel, mquintel@med.uni-goettingen.de

Published: 30 March 2009

Critical Care 2009, 13:126 (doi:10.1186/cc7738)

This article is online at http://ccforum.com/content/13/2/126

(c) 2009 BioMed Central Ltd

See related research by Zimmermann et al., http://ccforum.com/content/13/1/R10

\begin{abstract}
Extracorporeal lung-supporting procedures open the possibility of staying within widely accepted margins of 'protective' mechanical ventilation (tidal volume of less than $6 \mathrm{~mL}$ per $\mathrm{kg}$ of predicted ideal body weight and plateau pressure of less than $30 \mathrm{~cm} \mathrm{H}_{2} \mathrm{O}$ ) in most any case of respiratory failure or even of further reducing ventilator settings while still providing adequate gas exchange. There is evidence that, at least in some patients, a further reduction in tidal volumes might be beneficial. Extracorporeal procedures to support the lungs have undergone tremendous technical developments, thus reducing the procedure-related risks. However, what is true for ventilator settings should also be true for extracorporeal procedures: studies will have to demonstrate a convincing riskbenefit ratio. In addition, a simple reduction of the tidal volume will certainly not be the right answer. If extracorporeal support largely influences gas exchange, the 'optimal' tidal volume/positive endexpiratory pressure ratio keeping stress and strain low and avoiding alveolar derecruitment will still have to be individually defined.
\end{abstract}

In the previous issue of Critical Care, Zimmermann and colleagues [1] published a prospective observational study in which they applied a pumpless extracorporeal support system to improve gas exchange in a series of 51 patients still fulfilling acute respiratory distress syndrome (ARDS) criteria after 24 hours of 'advanced' respiratory support including low tidal volume ventilation $(<6 \mathrm{~mL} / \mathrm{kg})$ as well as positive end-expiratory pressure (PEEP) settings according to the ARDSNet 'high-PEEP' table. Positioning was performed either as prone positioning or as continuous lateral rotation therapy (CLRT), balanced volume therapy, and infection control $[1,2]$. The device consisted simply of two cannulas, a short tubing system, and an oxygenator. Such a system uses the individual cardiac output and resulting mean arterial blood pressure in an arteriovenous setting as the driving force. The principle is called pumpless ECMO (extra-corporeal membrane oxygenation), spontaneous arteriovenous carbon dioxide removal, or interventional lung assist, the last of which represents the 'official' labeling of the company providing the commercially available set used in this study. In brief, Zimmermann and colleagues were able to demonstrate efficient carbon dioxide removal at markedly reduced respiratory settings after 2 hours and even more so after 24 hours. The use of a well-defined algorithm including a restriction of the cannula size used for arterial cannulation decreased the number of ischemic complications when compared with two preceding studies of the same group in which a consistent rate of $10 \%$ ischemic complications was reported.

The study of Zimmermann and colleagues has evident limitations: lack of a control group and/or randomization, single-center performance, introduction and evaluation of several interventions at the same time, and, in addition, lack of clear definitions for terms like balanced volume therapy or infection control. Even so, this does not narrow the importance of these findings. On the contrary, looking at these data as a work in progress, we can draw several important conclusions.

First, no matter what kind of extracorporeal lung support is used, there is evidence that these procedures should represent well-defined steps in an escalating algorithm of acute lung injury/ARDS treatment. As stress triggered by mechanical ventilation is finally determined by the resulting transpulmonary pressures transmitted by each breath (whereas strain is related to the ratio between end-expiratory lung volume and the applied tidal volume), smaller tidal volumes in combination with an adequate level of PEEP (balancing the ratio between tidal volume and end-expiratory lung volume) should increase protectiveness of mechanical ventilation and ideally reduce mortality [3-5]. Recent experimental work by Dembinski and colleagues [6] demonstrated that a tidal 
volume reduction at the same level of PEEP led to a marked increase in shunt and low ventilation-perfusion $\left(V_{A} / Q\right)$ areas, indicating sustained derecruitment of the lungs. This phenomenon has also been demonstrated in the clinical setting by Richard and colleagues [7] in ARDS patients while reducing the tidal volume from 10 to $6 \mathrm{~mL}$. The application of lower tidal volumes obviously requires higher PEEP to avoid alveolar derecruitment. In addition, in the context of extracorporeal gas exchange, the weakness of the concept of setting PEEP according to a table becomes evident, and in the present study, what is very probably a 'false' PEEP reduction (obviously triggered by the increase in the $\mathrm{PaO}_{2} / \mathrm{FiO}_{2}$ [arterial partial pressure of oxygen/fraction of inspired oxygen] ratio after interventional lung assist insertion) was induced after 2 hours and had to be corrected after 24 hours.

Second, cannula size matters when an arterial vascular access is required. Even when the complication rate while using cannula sizes of less than $17 \mathrm{Fr}$ was reduced to $5.9 \%$ in the present study, it still remains high, possibly too high when this approach is used to achieve further 'protectiveness' during mechanical ventilation $[8,9]$. There is some evidence and personal experience that the restriction of cannula sizes to $15 \mathrm{Fr}$ or less minimizes the risk to a negligible level. Ideally, however, one venous double-lumen cannula would ensure the vascular access, and a small pump that is very easy to handle may be integrated into the system as a single-use product, delivering the flow in the range needed in a given case. For evident reasons, the simple rule 'the smaller, the better' is not true. Smaller tidal volumes require an individually adapted higher PEEP or continuous positive airway pressure (CPAP) level and a lot of experimental and clinical research to describe how an optimized individual tidal volume/PEEP setting can be defined while using a 'safe' extracorporeal lung support system that enables gas exchange.

\section{Competing interests}

$\mathrm{MQ}$ is a member of the advisory board of Novalung $\mathrm{GmbH}$ (Talheim, Germany) and therefore has received advisor honoraria. OM declares that he has no competing interests.

\section{References}

1. Zimmermann M, Bein T, Arlt M, Philipp A, Rupprecht L, Mueller T, Lubnow M, Graf BM, Schlitt HJ: Pumpless extracorporeal interventional lung assist in patients with acute respiratory distress syndrome: a prospective pilot study. Crit Care 2009, 13: R10.

2. Brower RG, Lanken PN, Maclntyre N, Matthay MA, Morris A, Ancukiewicz M, Schoenfeld D, Thompson BT; National Heart, Lung, and Blood Institute ARDS Clinical Trials Network: Higher versus lower positive end-expiratory pressures in patients with the acute respiratory distress syndrome. $N$ Engl J Med 2004, 351:327-336.

3. The Acute Respiratory Distress Syndrome Network: Ventilation with lower tidal volumes as compared with traditional tidal volumes for acute lung injury and the acute respiratory distress syndrome. N Engl J Med 2000, 342:1301-1308.

4. Terragni PP, Rosboch G, Tealdi A, Corno E, Menaldo E, Davini O,
Gandini G, Herrmann P, Mascia L, Quintel M, Slutsky AS, Gattinoni L, Ranieri VM: Tidal hyperinflation during low tidal volume ventilation in acute respiratory distress syndrome. $\mathrm{Am} J$ Respir Crit Care Med 2007, 175:160-166.

5. Grasso S, Stripoli T, De Michele M, Bruno F, Moschetta M, Angelelli G, Munno I, Ruggiero V, Anaclerio R, Cafarelli A Driessen B, Fiore T: ARDSnet ventilatory protocol and alveolar hyperinflation: role of positive end-expiratory pressure. $\mathrm{Am} J$ Respir Crit Care Med 2007, 176:761-767.

6. Dembinski R, Hochhausen N, Terbeck S, Uhlig S, Dassow C, Schneider M, Schachtrupp A, Henzler D, Rossaint R, Kuhlen R: Pumpless extracorporeal lung assist for protective mechanical ventilation in experimental lung injury. Crit Care Med 2007, 35:2359-2366.

7. Richard JC, Maggiore SM, Jonson B, Mancebo J, Lemaire F, Brochard L: Influence of tidal volume on alveolar recruitment. Respective role of PEEP and a recruitment maneuver. $\mathrm{Am} J$ Respir Crit Care Med 2001, 163:1609-1613.

8. Bein T, Prasser C, Philipp A, Muller T, Weber F, Schlitt HJ, Schmid FX, Taeger K, Birnbaum D: Pumpless extracorporeal lung assist using arterio-venous shunt in severe ARDS. Experience with 30 cases. Anaesthesist 2004, 53:813-819.

9. Bein T, Weber F, Philipp A, Prasser C, Pfeifer M, Schmid FX, Butz $B$, Birnbaum D, Taeger K, Schlitt HJ: A new pumpless extracorporeal interventional lung assist in critical hypoxemia/ hypercapnia. Crit Care Med 2006, 34:1372-1377. 